\title{
Implementation of Wireless ECG Acquisition System using Zigbee Technology
}

\author{
Prof. R. K. Moje ${ }^{1}$, Sujata R. Bhol' ${ }^{2}$, Monika U. Gote ${ }^{3}$ \\ Faculty, Department of Electronics, College of Engineering Manjari (Bk), Pune, India ${ }^{1}$ \\ Student, Department of Electronics, College of Engineering Manjari (Bk), Pune, India ${ }^{2,3}$
}

\begin{abstract}
The design and development of wireless ECG Acquisition system using zigbee technology has been developed and reported in this paper. This system is able to use for monitor physiological parameters such as ECG, heart rate, and human body temperature. This system is built up of electronic device which is worn on wrist and finger by a patient. Measuring vital signs with the help of several sensors, the patient is wirelessly monitored within hospital or his home. The device detects the heart rate, temperature this signals are send to a receiver unit. The receiver unit is connecting to the computer. This system is operated with the help of battery power. This low cost device can be help to monitoring the patient.
\end{abstract}

Keywords: Electrocardiogram (ECG), Sensor, Health monitoring, Zigbee.

\section{INTRODUCTION}

The heart is one of the most important organs in the entire human body. It is really nothing more than a pump. It is composed of muscle which pumps blood throughout the body, beating approximately 72 times per minute of our lives. The heart pumps the blood, which carries all the vital materials which help our bodies function and removes the waste products that we do not need. For example, the brain requires oxygen and glucose, which, if not received continuously, will cause it to lose consciousness. Muscles need oxygen, glucose and amino acids, as well as the proper ratio of sodium, calcium and potassium salts in order to contract normally.

The current status of heart disease in India is alarming, with suggesting that by the year 2020, the burden of cardiovascular diseases in India will surpass that of any other country in the world. It is estimated that17.5 million people die each year in India from cardiovascular diseases. $80 \%$ of all cardiovascular deaths are due to heart attacks and strokes. There are estimated 40 million heart patients in India. Out of which 19 million reside in urban areas and 21 million in rural areas. In India 175000 open heart surgeries per year happen. It is highest in the world. Factors responsible for heart problems are low-density lipoprotein cholesterol (LDL-C), diabetes, smoking, high blood pressure, genetic history, lifestyles, especially the higher intake of carbohydrate rich foods and lack of regular physical exercise etc.

The analysis of heart problem can be done using ECG waveform of the heart. ECG stands for Electrocardiography. It is the process of recording the electrical activity of heart using electrodes over a period of time. In existing system electrodes are placed on the skin or chest. These electrodes detect the small electrical changes on the skin that originate from heart muscles. It is commonly performed in cardiology test.

This system is designed as Wireless ECG acquisition system. In this system there is no need to attach electrodes to patient chest. Here we use wireless ECG sensor which can be attach to wrist of the patient. Noise is reduced as wireless sensor is used. The system consists of ECG sensor, heartbeat sensor, temperature sensor, LPC 2148, Zigbee module, and display unit.

\section{LITERATURE SURVEY}

- Currently, a number of studies have been proposed to address the issues of transmitting vital signs in nursing homes, homes and hospitals over wireless transmission. Patient monitoring systems are gaining their importance's the fastgrowing global elderly population increases demands for caretaking [1]. These systems use wireless technologies to transmit vital signs for medical evaluation.

- According to Kinsella and He's report from the US Census Bureau, the global elderly population is fast growing and will outnumber the population of children in near future. The aging society is bringing its impact on many developing countries and presents a stark contrast with the low fertility rate of these countries. The changes brought 


\section{ISO 3297:2007 Certified}

Vol. 5, Issue 4, April 2017

about by the aging society include an increasing demand for caretaking; thus, patient monitoring systems are gaining their importance in reducing the need for human resources [3]. Caretaking homes and hospitals have been planning on the use of biological sensors to effectively minister to their patients. Vital signs, such as body temperature, blood pressure, and sugar level, can be regularly collected and remotely monitored by medical professionals, achieving a comprehensive caretaking system.

- ZigBee is an open standard technology to address the demands of low-cost, low-power WMNs via short range radio [4].

- ZigBee is targeted at RF applications that require a low data rate, long battery life, and secure networking. Its mesh networking also provides high reliability and more extensive range. The ZigBee devices can be combined with WWANs to achieve a seamless platform of wireless patient monitoring. The ECG and heart rate data can not only help the caregivers to know the urgency of the fall-induced injury, but also show the probable reasons of falls. IEEE 802.15.4 is a standard which specifies the physical layer and media access control for low-rate wireless personal area networks (LR-WPANs) [5]. It is maintained by the IEEE 802.15.4 working group. It is the basis for the ZigBee.

- Varshney proposed a framework of patient monitoring systems, including patient monitoring devices, ad hoc wireless networks, and the receivers for healthcare professionals [6]. This framework uses four routing schemes (multicast, reliable multicast, broadcast, and reliable broadcast) and several enhancing schemes to improve the transmission reliability over wireless ad hoc networks.

- Jovanov et al. present wireless distributed data acquisition system. The system uses personal digital assistant as a mobile client to acquire data from individual monitors and synchronizes collected records with existing records on the telemedical server [9]. Each client device uses local flash memory as a temporary storage until reliable connection with a mobile client is established.

- Istepanian and Petrosian present an optimal zonal wavelet-based ECG data compression method, which reaches a maximum compression ratio of 18:1 with low-percent rms difference (PRD) ratios for a mobile telecardiology model [10]. The method also attains an ambulatory speed of up to $100 \mathrm{~km} / \mathrm{h}$ in urban channel profiles with a bit error rate of less than 10-15 and with an average reduction of $73 \%$ in the transmission time.

- Cypher et al. [8] surveyed previous work on wireless communications in support of healthcare networks. The authors only consider the case of one-hop transmission. From an analytical perspective, while using IEEE 802.15.4 standard for ECG, the maximum payload size only allows up to 118 samples per frame bringing the accumulation delay $236 \mathrm{~ms}$.

- Varshney and Sneha proposed protocols for power management under varying user densities, power levels, and numbers of hops to support a diversity of devices [7]. Their scheme provides a reliable message delivery at reasonable transmitted power.

- The use of patient operated ambulatory ECG recording equipment will normally be dependent on the patient's condition, as it requires a conscious patient to operate the equipment for making the ECG recordings.Patients undergoing acute myocardial infarction and subsequent arrhythmia leading to ventricular fibrillation and sudden cardiac arrest will not be able to operate such equipments. For a reliable monitoring system it is necessary to develop a completely automatic recording and analysing unit, which can detect cardiac abnormalities and automatically send alarm conditions to a central safety alarm system. It has been clearly documented that time is a critical factor in order to perform early cardiopulmonary resuscitation (CPR) and early defibrillation, and if the time to start CPR after a cardiac arrest increases, the chance of survival is reduced [11].

\section{BLOCK DIAGRAM}

This system is consisting of two sections, one is transmitter section and another one is receiver section.

\section{1) Transmitter Section}

The block diagram of the transmitter section shown in fig1.This transmitter section is connecting to the patientroom. Here we are using the following components,
1. ECG Sensor
2. Temperature Sensor
3. Heartbeat Sensor
4. LPC 2148
5. Power Supply
6. LCD
7. Zigbee Transmitter 


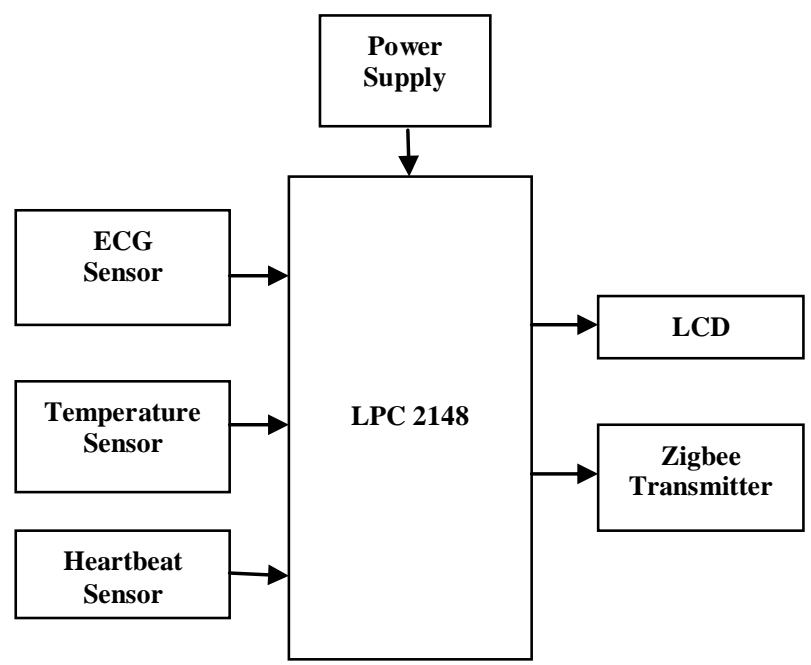

Fig. 1 Block Diagram of Transmitter Section

\section{ECG Sensor}

ECG Sensor is attached to the patient wrist. The signal obtained from the body is filtered and amplified. The sensor outputs an analog signal which is then converted by the analog to digital converter (ADC).

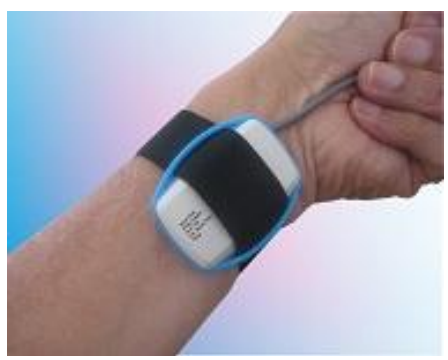

Fig. 2 ECG Sensor

Specifications of ECG sensor are as followed:-

- Frequency range: 0.05 to $16 \mathrm{~Hz}$

- Sampling rate: 470 samples/sec

- Battery/Supply: $3.6 \mathrm{~V}$

- Current drawn: 40mA

\section{Temperature Sensor}

In this system we have used LM35 temperature sensor. Which is simple three Pin sensor which directly calculate temp in Celsius. Amplified output is easily given by this temp sensor.

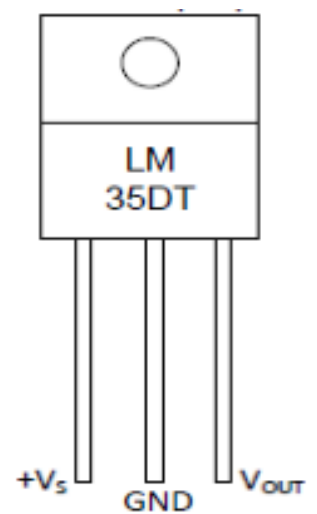

Fig. 3 Temperature Sensor 


\section{IJIREEICE

Specification of Temperature sensor is as followed:-

- It is of low cost.

- It does not need any calibration or trimming.

- Calibrated directly in Celsius (centigrade)

- $0.5^{0} \mathrm{C}$ Ensured accuracy $\left(\right.$ at $\left.+25^{0} \mathrm{C}\right)$

- Suitable for remote applications

- Operate from 4 to $30 \mathrm{~V}$

- Type: Analog

- Sensitivity: $10 \mathrm{mV}$ per degree Celsius

- Functional range: 0 degree Celsius to 100 degree Celsius

\section{Heartbeat Sensor}

Heart rate sensor can easily developed with using on IR led transmitter and receiver on same side of finger. The pulses and voltage sense by receiver is used for calculating heart rate. This is simple tip sensor attached to finger of patient.

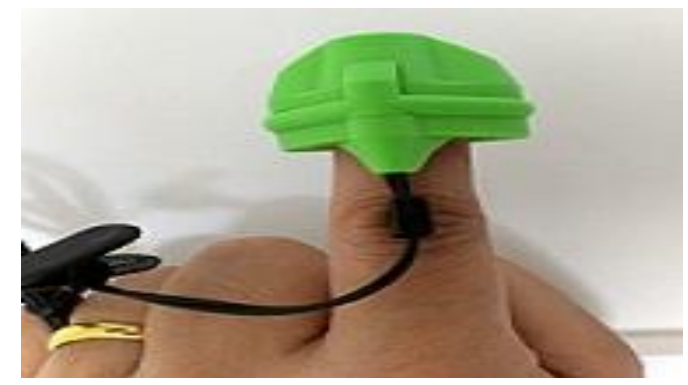

Fig. 4 Heartbeat Sensor

\section{LPC 2148}

In our system we are using LPC2148 as controller. It acts as the major controller unit of the system. Input from various sensors like ECG sensor, Heartbeat sensor, Temperature sensor, is given to this unit on which it process according to the programming and gives output to the zigbee transmitter. It needs $3.3 \mathrm{~V}$ to drive the ARM7 module.It has two 10 bit successive approximation analog to digital converterwith conversion time as low as $2.44 \mu \mathrm{s}$.

\section{Power Supply}

We have used bridge rectifier, filter, 7805 voltage regulator, LM 1117 IC for designing of power supply. It generates Constant 3.3V O/P and give it to drive of ARM module. On-board 9V 500mA transformer is used in which $230 \mathrm{~V}$ Primary and $9 \mathrm{~V}$ secondary is used.

\section{LCD}

We have used 16X2 characters liquid crystal display to display heartbeat count and body temperature of patient.

\section{Zigbee Transmitter}

Zigbee is an IEEE 802.15.4 based specification for a suite of high-level communication protocol. We take the data from LPC 2148and given to the zigbee transmitter. The physical range is approximate 10-20 meters. Zigbee transmitter transmits the ECG signal to the receiver section.

Zigbee operates in frequency range of $2.4 \mathrm{GHz}$. With hundreds of members around the globe, ZigBee uses the $2.4 \mathrm{GHz}$ radio frequency to deliver a variety of reliable and easy-to-use standards anywhere in the world.

The core Zigbee specification defines Zigbee's smart, cost-effective and energy-efficient mesh network. It's an innovative, self-configuring, self-healing system of redundant, low-cost, very low-power and even battery-free nodes that enable Zigbee's unique flexibility, mobility and ease of use.

\section{2) Receiver Section}

The block diagram of the transmitter section shown in Fig. 1 this receiver section is connecting to the doctor's control room.

Here we are using the following components,

1. Zigbee Receiver

2. PC 


\section{International Journal of Innovative Research in Electrical, Electronics, Instrumentation and Control Engineering}

ISO 3297:2007 Certified

Vol. 5, Issue 4, April 2017

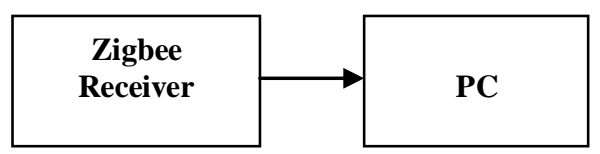

Fig. 5 Block Diagram of Receiver Section

\section{Zigbee Receiver}

Zigbee receiver receives the signal which is transmitted from zigbee transmmitter. It give output to the PC.

\section{2. $\quad$ PC}

PC is used to display ECG signal of patient. Zigbee module and PC are connected by using MAX232 IC. It converts signals from an RS-232 serial port to signals suitable for use in TTL compatible digital logic circuits.

\section{ADVANTAGES}

- It is used in military, sports and individual purpose.

- Low cost and wireless.

- Highly flexible

- Real-time application

- Reduces the human activity

- Quick response time

- Complete patient monitoring

- High accuracy

\section{CONCLUSION}

In this paper we present the monitoring of ECG and other body parameter which used widely in hospital and military. The advantages of this paper is using zigbee having high accuracy data transmission. This system is able to use for monitor physiological parameters such as ECG, heart rate, and human body temperature.

\section{REFERENCES}

[1] Shyr-Kuen Chen, Tsair kao, Chai- Tai Chan, Chih-Ning Huang, Chih-Yen Chiang, Chin-Yu Lai, A Reliable Transmission Protocol for ZigBee Based Wireless Patient Monitoring\| IEEE Trans Inf. Technol. Biomed.,vol.16,No.1 JANUARY 2012.

[2] S. Y. Lee, L. H.Wang, and Q. Fang, "A low power RFID integrated circuits for intelligent healthcare systems," IEEE Trans. Inf. Technol. Biomed., vol. 14, no. 6, pp. 1387-1396, Nov. 2010.

[3] K. Kinsella and W. He, An aging world: 2008,International Population Reports, U.S. Census Bureau, Washington, DC, Tech. Rep. P95/09-01, 2009.

[4] J. S. Choi and M. Zhou, Performance analysis of Zigbee based body sensor networks, in in Proc. IEEE Conf. Syst., Man Cybern.,2010, pp. 24272433.

[5] Jin-Shyan Lee, Yu-Wei Su, and Chung-Chou Shen Hsinchu, Taiwan A Comparative Study of Wireless Protocols: Bluetooth, UWB, ZigBee, and Wi-Fi,\| The 33rd Annual Conference of the IEEE Industrial Electronics Society (IECON) Nov. 5-8, 2007, Taipei, Taiwan.

[6] U.Varshney, Improving wireless health monitoring using incentive-based router cooperation,\| Computer, vol. 41, pp. 56-62, May 2008

[7] U. Varshney and S. Sneha, Patient monitoring using ad hoc wireless networks: Reliability and power management,\|lEEE Commun. Mag., vol. 44, no. 4, pp. 49-55, Apr. 2006.

[8] D. Cypher, N. Chevrollier, N. Montavont, and N. Golmie, -Prevailing over wires in healthcare environments: benefits and challenges, $\|$ IEEE Commun. Mag., vol. 44, no. 4, pp. 56-63, Apr. 2006.

[9] E. Jovanov, A. O’Donnel, A. Morgan, B. Priddy, and R. Hormigo, - Prolonged telemetric monitoring of heart rate variability using wireless intelligent sensors and a mobile gateway, in Proc. Eng.Med. Biol., 24th Annu. Conf. Annu. Fall Meet. Biomed. Eng. Soc. EMBS/BMES Conf., 2002. Proc. 2nd Joint, oct. 2002, vol. 3, pp. 1875-1876.

[10] R. S. H. Istepanian and A. A. Petrosian, -Optimal zonal wavelet-based ecg data compression for a mobile telecardiology system, IEEE Trans. Inf. Technol. Biomed., vol. 4, no. 3, pp. 200-211, Sep. 2000

[11] T. J. Bunch, R. D. White, B. J. Gersh, R. A. Meverden, D. O. Hodge, K. V. Ballman, S. C. Hammill, W. K. Shen, and D. L. Packer, "Long-term outcomes of out-of-hospital cardiac arrest after successful early defibrillation," New England journal of medicine, vol. 348, pp. 2626-33, 2003.

[12] E. C. Gullette, J. A. Blumenthal, M. Babyak, W. Jiang, R. A. Waugh, D. J. Frid, C. M. O’Connor, J. J. Morris, and D. S. Krantz, “Effects of mental stress on myocardial ischemia during daily life," Amer. Med. Assoc., vol. 277, pp. 1521-1526, 1997. 